\title{
La creatividad en el contexto educativo: adiestrando capacidades
}

\author{
Jesús Alberto Valero Matas \\ Coordinador del GIR Trans REAL Lab de la Universidad de Valladolid \\ y profesor titular de Sociología de la Facultad de Educación de Palencia \\ valeroma@soc.uva.es
}

Este trabajo ha sido seleccionado para su publicación por: don Alfonso Aguiló Pastrana, doña Milagros Antón López, don Fernando Checa García, don Ángel de Miguel Casas, doña Laura Rayón Rumayor y don Javier Manuel Valle López.

\section{Extracto}

Desde hace algún tiempo la creatividad se ha convertido en un fenómeno indispensable en la educación. Como ocurre con todo aquello que emerge de manera impulsiva, se ha transformado en un factor esencial en los procesos de aprendizaje. También se le ha atribuido el rol de gurú, puesto que la creatividad se ha convertido en la solución de las capacidades, destrezas y habilidades con las que hay que dotar a los estudiantes. Con este trabajo lo que se pretende destacar fundamentalmente es que la creatividad es importante, pero que es necesario realizarla en un contexto y espacio adecuados. No sirve simplemente con decir «se hace creatividad", sino que es importante explicar "cómo se hace» y «para qué se hace». Por tanto, este artículo es una reflexión crítica sobre esta necesidad que es la creatividad, algo que se ha transformado en un fenómeno dentro de nuestro sistema educativo. Por otra parte, es cierto que la educación necesita creatividad, pero no todo responde a los parámetros de la misma; en la actualidad existen muchos críticos con los modelos de aprendizaje educativos al sostener que la escuela mata la creatividad, así como hay otros críticos contrarios a esta idea.

Palabras clave: educación; talento; capacidad; formación.

Fecha de entrada: 03-05-2018 / Fecha de aceptación: 10-07-2018 / Fecha de revisión: 15-03-2019

Cómo citar: Valero Matas, J. A. La creatividad en el contexto educativo: adiestrando capacidades. Tecnología, Ciencia y Educación, 13, 150-171. 


\title{
Creativity in the educational context: training skills
}

\author{
Jesús Alberto Valero Matas
}

\section{Abstract}

The creativity has become an indispensable phenomenon in education for long time. As everything that emerges impulsively, it has become an essential factor in learning processes. Creativity has been attributed the role of guru, since it has become the solution of the abilities, skills and abilities that must be provided to students. This work tries to emphasize fundamentally the importance of the creativity, although, it is necessary to develop it in a suitable context and environment. It does not serve simply to say "creativity is made", but it is important to explain "how it is done" and "what it is done for». Therefore this article is a critical reflection on this need that is creativity, something that has become a phenomenon within our educational system. On the other hand, undoubtedly, education needs creativity, not everything shown as creativity is creativity. Currently there are many critics of educational learning models because they say that the school kills creativity, as well as there are others contrary to this opinion.

Keywords: education; talent; capacity; training.

Citation: Valero Matas, J. A. Creativity in the educational context: training skills. Tecnología, Ciencia y Educación, 13,150-171 


\section{Sumario}

1. Introducción

2. ¿Qué es la creatividad?

2.1. ¿Ser creativo es innato o se construye?

2.2. ¿La creatividad se puede aprender?

2.3. ¿Cómo podemos adiestrar la mente creativa?

3. Teorías sobre la creatividad

3.1. Teoría del asociacionismo

3.2. Teoría gestáltica

3.3. Teoría existencialista

3.4. Teoría factorial

3.5. Teoría de las inteligencias múltiples

3.6. Teoría humanística

4. Creatividad y educación

4.1. Algunas indicaciones metodológicas para desarrollar la creatividad desde la educación

5. A modo de reflexión

Referencias bibliográficas

Nota: este trabajo ha sido posible al proyecto «Analizar las necesidades y demandas de los estudiantes de primaria y secundaria en la enseñanza STEM», que se enmarca dentro de la Convocatoria de Ayudas a Proyectos de I+D+i 2017 de la Fundación Hergar (categoría: Aplicación de las TIC a la Educación de Adultos y a las Ciencias de la Salud). 


\section{Introducción}

En este artículo se intenta poner de relieve un aspecto importante, que es situar la creatividad en el lugar que le corresponde y que esta no sea considerada como la «respuesta salvadora» a las «debilidades» de planificación del sistema educativo.

A raíz de varias publicaciones, algunos centros educativos, así como varias organizaciones empresariales han recurrido al aprendizaje basado en inteligencias múltiples. No obstante, en relación a este tipo de aprendizaje existen críticos para quienes los resultados que se obtienen no son tan impactantes y que piensan que este aprendizaje puede tener un efecto negativo. Recordemos aquellos test que se hacían en los colegios para definir el futuro de los estudiantes. Según estos test, muchos alumnos no servían para estudiar y se les aconsejaba que se formaran en un oficio. Sin embargo, una gran cantidad de estudiantes hicieron caso omiso de los resultados de estos test y terminaron estudiando y trabajando como médicos, abogados, profesores o ingenieros, entre otros.

Ante temas tan importantes como la educación no se puede y no se debe jugar a «ensayo» $y$ «error». Es necesario analizar la situación y diseñar programas que respondan a necesidades educativas, sociales y laborales.

\section{2. ¿Qué es la creatividad?}

La creatividad se refiere a la capacidad de crear de una persona. Consiste en encontrar procedimientos o elementos que nos permitan realizar labores de forma distinta a la habitual para lograr un determinado objetivo. Lo que se intenta es buscar soluciones originales.

Una persona creativa tiene confianza en sí misma, una buena intuición, gran imaginación e ilusión y, sobre todo, curiosidad por descubrir cosas nuevas. El reto está en hacer que todas esas capacidades se desarrollen por los niños en la escuela a través de proyectos o sistemas innovadores.

Está claro que la creatividad no es algo nuevo, pues es una parte del intelecto humano y, por ende, desde siempre ha existido. Remontémonos a clásicos como Pitágoras, Arquímedes, Homero, Sófocles y Esquilo, entre otros. O a personajes más modernos, como Dante, Leonardo da Vinci, Cervantes, Einstein o Hawking. En todos ellos encontramos creatividad. A pesar de esto, hasta tiempos recientes la creatividad no ocupó un lugar importante en la investigación. Y, desde entonces, muchas han sido las definiciones y aportaciones 
que se han dado sobre este concepto. En un principio, la psicología fue la más prolífica en la definición del concepto de «creatividad». Posteriormente, la educación tomó el remplazo y entró de lleno en el empleo de dicho concepto, hasta el punto de que diseñó y sigue proyectando programas educativos centrados en la creatividad.

Entre las definiciones más destacadas sobre este concepto encontramos la de Guilford (1950), para quien la creatividad se refiere a las aptitudes que son características de los individuos creadores, como la fluidez, la flexibilidad, la originalidad y el pensamiento divergente. En Torrance (1965) se define como «un proceso que vuelve a alguien sensible a los problemas, a las deficiencias, a las grietas o a las lagunas en los conocimientos y lo lleva a identificar dificultades, a buscar soluciones, a hacer especulaciones o a formular hipótesis, a probar y comprobar estas hipótesis, a modificarlas si es necesario, además de comunicar los resultados» (p. 36). Csikszentmihalyi (1998) transforma dicho concepto en cualquier acto, idea o producto que cambia un campo ya existente, o que modifica un campo ya existente en uno nuevo, y Gardner (1995) expresa que no es una especie de fluido que pueda manar en cualquier dirección. «La vida de la mente se divide en diferentes regiones, que yo denomino "inteligencias", como la matemática, el lenguaje o la música. Y una determinada persona puede ser muy original e inventiva, incluso imaginativa, en una de esas áreas, sin ser particularmente creativa en ninguna de las demás» (p. 98).

Estas definiciones responden a un proceso marcado por la evolución y el uso del concepto en periodos de tiempo determinados. La primera de ellas marca un momento inicial que posteriormente, a medida que vaya desarrollándose y siendo más recurrente, irá cambiando.

La creatividad como esencia humana y social ha sido bastante cuestionada, puesto que algunos piensan que se trata de un tema innato al ser humano y otros que puede ser aprendida. Intentaremos desvelar sus atributos para más adelante analizar la relación creatividad-educación.

\section{1. ¿Ser creativo es innato o se construye?}

Como se ha comentado anteriormente, muchos consideran la creatividad como algo inscrito en el sujeto, es decir, se nace creativo o no. Sin embargo, algunos psicólogos, como Fromm, creen que la creatividad puede desarrollarse o fomentarse mediante el aprendizaje. Fromm decía que la creatividad no es una cualidad característica particular de los artistas o de las personas relacionadas con el arte, sino una actitud que puede poseer cualquier persona.

Atendiendo a esto, todos tenemos la capacidad de crear, y, por lo tanto, todos somos creativos, si bien, unos más en esencia que otros

La creatividad puede desarrollarse o fomentarse mediante el aprendizaje. Trabajando y adecuando la creatividad se puede lograr una destreza creativa 
(Gardner, 1995). Algunos autores juegan con el hecho de que, durante la infancia, los seres humanos intentan pintar, hacer construcciones con piezas, tocar un instrumento, etc.

Por lo tanto, como ocurre con otros aspectos del ser humano, la creatividad, si se cultiva, se puede desarrollar, es decir, trabajando y educando la creatividad, se puede lograr una destreza creativa; de otra manera, no se logrará una competencia creativa a no ser que, como se ha comentado con anterioridad, sea innata. En esta línea encontramos las aportaciones psicológicas de Kris (1952), quien postula que la creatividad es una habilidad que permite la conexión de unas ideas con otras. En definitiva, alguien es creativo si mediante el aprendizaje logra conectar las ideas entre sí. Getzels y Jackson (1962) separan la inteligencia y la creatividad. Según estos autores la inteligencia se mueve en espacios estereotipados y, en función de esta, se educa en las escuelas, mientras que la creatividad se aleja de los estereotipos, crea criterios propios y no se enseña en las escuelas. Por no extendernos, Parnes (1967) consideraba que la creatividad podía ser entrenada mediante múltiples técnicas, tácticas y estrategias. Para él, la creatividad no era más que la capacidad que el individuo debe entrenar para encontrar vínculos entre ideas no relacionadas con anterioridad y expresadas en nuevas experiencias o productos. Esto nos conduce al siguiente interrogante.

\section{2. ¿La creatividad se puede aprender?}

Para muchos teóricos de la psicología, la creatividad se identifica con el pensamiento creativo o divergente, y, por lo tanto, se identifica con la capacidad del ser humano para procesar la información que ha sido percibi-

Para muchos teóricos, la creatividad se identifica con el pensamiento creativo o divergente da (Valero-Matas, Valero-Oteo, Coca y Leyva, 2016). En consecuencia, estos autores la catalogan en el mismo nivel que otras capacidades del cerebro, como son la inteligencia o la memoria, de ahí que su concepción de ser aprendida se apoye en que tanto la inteligencia como la memoria se desarrollan a lo largo de la vida.

A raíz de estas ideas han surgido centros especializados en potenciar la habilidad creativa mediante técnicas y ejercicios que ayudan a activar la capacidad creadora con la que se nace. Bajo el lema de la «creatividad como motor del mundo», estos centros dicen potenciar la capacidad creativa que está dentro del sujeto. Robinson (2009) expresa que la creatividad se activa si uno mantiene vivos cuatro elementos:

- Dar con el elemento, es decir, encontrar nuestra habilidad natural.

- Conectar con la pasión. En otros términos, dar con la actividad que nos es casi innata. 
- Capacidad de control. En sus propias palabras, es poder controlar los materiales y elementos que nos interesan.

- Todo el mundo puede ser creativo en un ambiente adecuado.

Robinson concluye hablando de lo que podríamos etiquetar como un quinto aspecto: llevar a cabo una etapa divergente y ser críticos con nuestras ideas. De aquí surge un tercer interrogante, que pasamos a explicar en el siguiente apartado.

\section{3. ¿Cómo podemos adiestrar la mente creativa?}

Los diseñadores de programas de activación y aprendizaje de la creatividad sostienen que la mente creativa debe ser estimulada desde los primeros años del niño y hay que seguir cultivándola a lo largo de la vida. Aquí juegan un papel primordial los dos primeros agentes de socialización de los niños: la familia y la escuela. Ambos agentes deben potenciar la creatividad.

A los niños les resulta sencillo aprender a ser creativos si se les potencia el entusiasmo por la indagación o la exploración. Tanto los padres como los docentes deben hacer ver o mostrar que un problema o circunstancia puede tener diferentes soluciones. La escuela debe ofrecer a

La escuela debe ofrecer a los niños actividades que premien la innovación y en las que se acepten los errores y fracasos los niños actividades que premien la innovación y en las que se acepten los errores y fracasos, y en este ámbito es donde hay que estimular a los niños a que rompan con los juegos estereotipados y creen otros nuevos. No obstante, a medida que se crece biológica e intelectualmente, la crítica puede frustrar la creatividad, por ello, es necesario que los niños aprendan a expresar y a recibir críticas constructivas, y a saber discernir las críticas positivas de las negativas.

A modo de resumen, todo esto pone de relieve que en un futuro no muy lejano, en cualquier ámbito de la vida cotidiana, la creatividad estará presente y será necesaria, especialmente en las relaciones personales y en el desempeño laboral.

\section{Teorías sobre la creatividad}

\subsection{Teoría del asociacionismo}

Esta teoría se fundamenta en el pensamiento aristotélico, que después adoptarán los filósofos británicos Locke y Hume, quienes sostenían que el principio de asociación era 
aplicable a todos los procesos mentales y que las ideas derivadas de la mente estaban asociadas a leyes naturales, especialmente a la ley de continuidad y de semejanza. Posteriormente, esta teoría será más potenciada por Hartley y Stuart Mill, cuya tesis se basaba en la combinación de estímulos y elementos captados por los sentidos.

Uno de los máximos exponentes de esta teoría es Mednick (1962), quien postula que la creatividad es interpretada como una transformación de los elementos asociativos, dando lugar a la creación de nuevas combinaciones ante situaciones de demanda concretas o ante una utilidad. Este autor continúa diciendo que cuanto más remotas sean las ideas del nuevo producto/hecho, más creativo es el proceso o la solución. Aquí intervienen principalmente dos factores:

- La cantidad de elementos que el sujeto domina.

- Las asociaciones que el sujeto establece entre dichos elementos.

A su juicio pueden producirse tres tipos de asociaciones creativas:

- Serendipity. Logro de asociaciones mediante el hecho causal de una contigüidad de perfiles que conducen a nuevos descubrimientos.

- Semejanza. Reunión de palabras, sonidos, estructuras, objetos, mayoritariamente empleados para la creación artística.

- Mediación. La asociación entre símbolos que pueden suscitar nuevas ideas, como acontece en las ciencias experimentales, y que requieren de mayor abstracción que otras.

Mednick (1962) creó un test para medir el asociacionismo de ideas que denominó «test de asociaciones remotas» (RAT). Sin embargo, es un medidor que no tiene presente muchos aspectos, entre los que destaca la motivación; y, por otro lado, es demasiado simple resolver la creatividad mediante la asociación de ideas con lo complejo que es el ser humano.

\subsection{Teoría gestáltica}

Esta corriente encuentra una gran analogía entre el proceso de pensar creativo y el proceso perceptivo del individuo. El sujeto piensa estructurando, reorganizando y agrupando sus ideas, de manera que, gestionando estas ideas sistematizadas, se llega a la resolución de problemas. Por lo tanto, el proceso será más creativo y el producto más innovador cuanto más pronunciada es la mudanza entre las diferentes conexiones. Esta corriente toma algunas ideas de la teoría asociativa. 
La teoría gestáltica de la creatividad se proyecta sobre la apertura a lo nuevo, es decir, al considerar que creatividad y producto son sinónimos, esto implica la necesidad de aportar ideas nuevas, desde un planteamiento abierto para la resolución de problemas. El sujeto debe aprender a ver desde diferentes dimensiones el problema, cambiando la forma tradicional de afrontarlo.

La teoría gestáltica define la creatividad como una acción por la que se produce o moldea una idea o visión. Esa novedad surge repentinamente porque es producto de la imaginación, y no de la razón y la lógica.

\subsection{Teoría existencialista}

La creatividad solo es posible cuando el individuo encuentra su mundo, el de su entorno y el de sus semejantes. Según sea la intensidad del encuentro con el mundo circundante, su grado de creatividad será uno u otro.

May (1977) fundamenta la creatividad en el encuentro. En otras palabras, el sujeto y el entorno se encuentran, y el objeto debe ser observado y asimilado por el sujeto. Los medios que utiliza para expresar esa vivencia son secundarios. Lo importante es el encuentro.

Para los existencialistas, en el ser humano existe una lucha existencial entre dos impulsos: el de permanecer abierto al entorno y el de mantenerse cercano. Los existencialistas entienden la creatividad como el triunfo de un ser abierto, captador, vigilante sobre la perspectiva íntima, incorporada a lo habitual, cerrada.

\subsection{Teoría factorial}

Guilford (1988) diferencia entre las personas que son creativas y las que no lo son. Desarrolla la teoría intelectual de la creatividad, según la cual el individuo creativo está motivado por el impulso intelectual de estudiar los posibles problemas y encontrar soluciones a los mismos.

La creatividad, según tus tesis, conlleva fluidez, flexibilidad y originalidad. Si no existe esto, una persona difícilmente podrá ser creativa. Las personas que son fluidas, en poco tiempo, producen múltiples ideas que ayudarán a resolver muchos problemas.

A Guilford no le importa el campo en el que se desarrolla la creatividad, pues la considera como un simple elemento de aprendizaje, y aprender es captar nuevas informaciones o establecer relaciones nuevas con informaciones viejas. Todo aprendizaje contiene un aspecto general, transmisible a otros cometidos de carácter general, y otro específico, no transmisible. La transmisión es por semejanza. Este autor diferencia entre pensamiento «Convergente» $y$ «divergente». 
Torrance y Myers (1996) elaboró un test para medir el grado de creatividad de las personas. También argumentó que una persona que posee las habilidades propias del pensamiento creativo no quiere decir que posea una conducta creativa, sino que posee un potencial creativo.

\subsection{Teoría de las inteligencias múltiples}

Según el pensamiento de Gardner (1987), el individuo creativo es una persona que resuelve problemas con regularidad, elabora productos o define cuestiones nuevas en un campo, de un modo que al principio es considerado nuevo, pero que al final llega a ser aceptado en un contexto cultural concreto. Gardner (2011) afirma que las soluciones creativas a los problemas se dan con mayor frecuencia si los individuos se dedican a una actividad por puro placer que cuando lo hacen por recompensas o por exigencias exteriores. Saber que uno será juzgado como creativo limita las posibilidades creativas.

Gardner (2012) considera que es posible fomentar la creatividad. En ese sentido, la concepción propuesta va más allá de la idea de creatividad como capacidad de pensamiento divergente, para incorporar la consideración de otros factores necesarios con los que ese pensamiento divergente acabe fructificando en la actividad creativa, como son la relación del individuo con el sistema simbólico y la disciplina, a los que contribuye, y la relación del individuo con su contexto social próximo, aportando así una mayor complejidad, necesaria para el análisis de la creatividad.

Por otra parte, estima que la creatividad es un fenómeno multidisciplinario y multifuncional. Entiende que el estudio de la creatividad, por su propia naturaleza, se centre más en los factores personales y haga uso de las perspectivas biológica, epistemológica y sociológica para hacer un análisis de conjunto.

En su análisis de la creatividad del individuo, Gardner (2011) establece cuatro aspectos que hay que tener presentes:

- La persona creativa lo es en un área determinada y no es creativa en todos los campos.

- La creatividad se extiende a un amplio elenco de aspectos, desde la elaboración de productos hasta la resolución de problemas.

- La persona creativa lo expresa de manera continuada y, en ese aspecto, no es una cuestión de pura coincidencia.

- La persona será creativa cuando exista un reconocimiento por parte de otras personas acerca de su creatividad; de lo contrario, será un creativo en potencia.

Atendiendo a ese carácter dado a la creatividad de multidimensional y multifuncional, Gardner resalta tres elementos en su sistema creativo: 
- Individuo. La importancia de que el creador recurra a la cosmovisión del niño pequeño combinándolo con la esfera del ser adulto, ya seguro de sí mismo.

- Trabajo. Responde al tópico o disciplina donde el individuo destaca como creativo. Mientras desarrolla su trabajo recurre a un amplio elenco de símbolos que son parte de su mundo creativo, que llevan al individuo a revisar los existentes o a imaginar otros nuevos.

- Ambiente humano. La relación entre el individuo y otras personas es muy importante en el proceso creativo. Aunque se piensa que algunas personas creativas trabajan aisladas, la presencia de otros individuos es fundamental en dicho proceso. Este elemento es importante para Gardner (2011), porque diferentes agentes ejercen influencia en el desarrollo y posterior habilidad creativa. Como él mismo señala, la familia, los docentes o las personas que han ayudado o rivalizado en el momento del crecimiento creativo son determinantes.

\subsection{Teoría humanística}

Maslow (1994) sostiene que en todo ser humano existe una tendencia a la creatividad, algo propio de su naturaleza y que no está sujeto a ninguna planificación ni a objetivos planificados. Como el acto creativo es estimulante y gratificante, esto hace que el sujeto siga activando la creatividad. En otros términos, con la creatividad el sujeto se autorrealiza. Una persona con mucha creatividad tiende a realizar las cosas de manera más fácil y espontánea.

Maslow diferencia entre distintos tipos de creatividad:

- Creatividad primaria. Está asociada al numen creador y se caracteriza por la espontaneidad y la improvisación.

- Creatividad secundaria. Tras haber conseguido la inspiración, la creatividad se plasma en un producto, ya sea un cuadro, una novela, un artefacto, etc. Requiere grandes dosis de preparación y esfuerzo.

- Creatividad integrada. Es aquella que requiere un don especial y mucho estudio, conocimiento y concentración para alcanzar la perfección creadora.

Lo que propone la teoría humanística es que la creatividad está sujeta al ambiente de la experiencia, de la autorrealización y de los cambios sociales.

\section{Creatividad y educación}

Desde los años noventa del siglo pasado, la creatividad y su inclusión en la educación han adquirido bastante importancia, hasta tal punto que muchos colegios anuncian en su 
publicidad «educación basada en la creatividad». A raíz de esto, un amplio grupo de teóricos han elaborado diversas proyecciones educativas que parten de la creatividad. Dichas aportaciones surgen desde perspectivas diferentes, bien porque están orientadas al sujeto creativo o al transcurso u objetivo del proceso creativo.

Pero ha sido en el siglo XXI cuando la educación y la creatividad se han puesto en acción con el objetivo de introducir un cambio en el modelo educativo estandarizado. Se está fomentando el discurso de que en el mundo en el que vivimos se necesitan personas más creativas, innovadoras, habilidosas, astutas, etc., pero el sistema educativo que existe no fomenta esto, sino individuos que son como máquinas (habilidosos en los exámenes, estigmatizados ante el fracaso y el error, etc.), argumentando la pérdida de la interacción entre emociones y mente, confundiendo pseudoconocimiento con sabiduría.

Las aportaciones de Robinson en The Element (2009) extendieron la dinámica de la creatividad como forma de mejora de la educación bajo el prisma de la creatividad. En su obra, este autor expone que el actual sistema escolar anula la capacidad creativa de los niños y, en consecuencia, es necesario transformar las escuelas, es decir, cambiar el sistema educativo estandarizado por uno innovador, basado en la creatividad.

Para el británico, todas las personas cuentan con una gran capacidad creativa, pero el sistema anula esa tendencia. Según él, todos los niños (para nosotros no todos) sienten predisposición al riesgo, a abrazar lo nuevo, no tienen miedo al error, etc. A medida que van creciendo y cambiando de centros educativos, los niños son canalizados hacia lo corriente, lo establecido, etc., y se les inculca el miedo a la equivocación y la frustración. En palabras de este autor, se los educa sin tener en cuenta su capacidad creativa. Además, continúa diciendo, todo su conocimiento se focaliza en una respuesta única; es decir, aunque un mismo problema puede ser abordado desde diferentes puntos de vista y, por ende, tener múltiples soluciones, en la escuela solo se admite una, y es la del docente; el resto son soluciones erróneas.

Atendiendo a estas nuevas tendencias o propuestas, educar en la creatividad implica enseñar para la transformación y formación de personas que sean fecundas en aspectos como la originalidad, la flexibilidad, la proyección de futuro, la iniciativa, la confianza, la valentía ante nuevos retos, así como que estén capacitadas y adiestradas para afrontar problemas y un sinfín de nuevas realidades a lo largo de su proyecto de vida. Como decía Amabile (1996), la creatividad también dota de los mecanismos necesarios para innovar y crear en la sociedad del siglo XXI.

Cuando los educadores hablan de educar en la creatividad, la mayoría lo enfocan hacia el desarrollo personal en los discentes y hacia la mejora profesional de la práctica educativa. Todo ello dentro de un contexto histórico-social orientado a la integración educativa, sabiendo, como se ha indicado en los apartados anteriores, que la mayoría de los teóricos entienden la creatividad como aquella actitud que permite a las personas tener una actitud más flexible y renovadora. Con la creatividad en el campo educativo se logra que los discentes y docentes eli- 
minen aquellos obstáculos que impidan construir una escuela de futuro cimentada en la integración, la solidaridad, el respeto, la reflexión, lo divergente, el desarrollo, la apertura, etc.; es decir, una escuela basada y centrada en abarcar todas y cada una de las necesidades de todos los estudiantes.

En las etapas iniciales del proceso educativo, los docentes expresan la necesidad de educar en la creatividad, proyectando un aprendizaje centrado en la capacidad de ver la realidad con
Con la creatividad en el campo educativo se logra que los discentes y docentes eliminen aquellos obstáculos que impidan construir una escuela de futuro cimentada en la integración, la solidaridad, el respeto, la reflexión, lo diferente, el desarrollo, la apertura, etc. la mirada abierta y saber dar respuestas de manera diferente. Los educadores de niños pequeños emplean cinco palabras en la transmisión de la creatividad en la educación: por un lado, reflexión y tiempo, aspectos destacados en el desarrollo diario en el aula; por otro, esfuerzo, porque es una herramienta de perseverancia (no rendirse ante las adversidades), motiva al estudiante, le ilusiona con lo que está haciendo y tiene una recompensa, intrínseca y extrínseca; finalmente, el error y el fracaso, conceptos poco aceptados por la mayoría de las culturas o, mejor dicho, poco practicados. Una de las principales ideas propuestas anteriormente es la educación o el aprendizaje del error, es decir, si nos confundimos, lo volvemos a intentar, sin que ello menoscabe nuestra personalidad y deprima al estudiante.

Conjugando el modelo sociológico de la creatividad con el proceso creativo-educativo se obtiene un paradigma edificado en torno a tres elementos:

- Creatividad adquisitiva-formativa. Es la creatividad que se consigue mediante el proceso de aprendizaje formativo. Las personas con poca creatividad pueden desarrollarla por medio de diferentes técnicas, instrumentos, etc.

- Creatividad elaborativa. Hace referencia a la creatividad que el individuo va moldeando, construyendo o dirigiendo, bien porque la tiene de manera innata, o bien porque la adquiere a través del proceso de socialización o mediante el aprendizaje formativo. Como consecuencia de todo esto, el individuo va confeccionando una creatividad ajustada a las necesidades de cada momento, de manera que le ayude a hacer frente a las necesidades y/o problemas surgidos en esa coyuntura.

- Creatividad proyectiva. Es la creatividad programada por la persona en pro de satisfacer, mejorar o ayudar a la sociedad. Esta se verá influida por el resto de creatividades, porque su fin último es lograr un mundo mejor.

De todo esto se deduce que el marco teórico-práctico referido a la formación requiere planificación y programación. Este modelo establece diferentes etapas de evolución social que se planificarán y programarán amparándose en diversos indicadores: capacidad, situación, profesión o etapa formativa; y cada uno requerirá técnicas, instrumentos y metodologías 
propias. Así, cabe esperar que el docente que trabaje creativamente no se convierta en un simple consumidor de conocimientos científicos; para ello, debe utilizar técnicas y herramientas educativas que le conduzcan a crear situaciones docentes concretas que potencien la creatividad dentro de un marco de acción individual-colectiva, evitando ahogar la capacidad de producción innovadora. De lo contrario, la creatividad de la construcción quedará reducida a la creatividad de la destrucción.

La creatividad puede ser innata o educada. De Bono (1999) dice al respecto:

La creatividad no es una cualidad o destreza cuasi mística. Tampoco es una cuestión de talento natural, temperamento o suerte, sino una habilidad más que podemos cultivar y desarrollar. Obviamente, si se tratara de una condición natural, no tendría sentido el esfuerzo para cultivarla y mejorarla, y si no fomentáramos la capacidad creativa, esta dependería en todo del talento natural (p. 24).

Por lo tanto, tanto en un caso como en otro se debe propiciar el espacio para cultivarla. Ahora bien, la educación de la creatividad profesional es una tarea compleja y multifactorial. La creatividad del docente debe estar dirigida a determinar y descubrir contradicciones propias del ejercicio de la profesión en condiciones cambiantes, de ahí que el proceso de trabajo de la creatividad profesional en este ámbito esté determinado por problemas profesionales y comience cuando el docente pone al estudiante ante las contradicciones, de forma tal que se produzca la toma de conciencia del problema y surja en él la necesidad de resolverlo.

\section{La creatividad docente consiste en la comprensión lógica de tareas, actividades y procedimientos para la resolución de problemas}

La creatividad docente consiste en la comprensión lógica de tareas, actividades y procedimientos para la resolución de problemas; cuestión que requiere de la implicación de los estudiantes en el proceso creativo. Ante este hecho es necesario ser conscientes de que no se está ante la lógica clásica, sino frente a una lógica abductiva, la lógica compleja o la lógica divergente.

Una manera de que los estudiantes se sientan atraídos hacia la participación descansa, como dice Landau (1987), en explicar «a los escolares que es necesaria una determinada actitud para el aprendizaje creativo: el alumno debe saber que de él se espera creatividad» (p. 112). Teniendo predisposición al aprendizaje creativo se consigue generar un entorno dinámico en el que el estudiante no está sujeto solo a la transmisión de conocimientos, sino que se encuentra en un clima dinámico donde aflora la curiosidad, la originalidad, la novedad, la imaginación y la reflexión. La apertura a estas dinámicas de aprendizaje, junto a la transmisión de conocimientos, destrezas, habilidades, etc., puede resultar infructuosa si no existe un verdadero reconocimiento de la importancia y del valor que tiene la creatividad en el individuo como ser social. De no ser así, será difícil alcanzar los objetivos deseados. 
El proceso docente estimula la creatividad siempre que haya conciencia de esa demanda y disponibilidad epistémica y práctica de los recursos didácticos necesarios para lograrlo mediante diferentes formas organizativas del proceso de enseñanza-aprendizaje. Como propone De la Torre (2009), «la creatividad docente se manifiesta en la propuesta de objetivos didácticos, en las actividades de aprendizaje, en la evaluación, pero sobre todo en la metodología utilizada» (p. 162). Una de estas metodologías potenciadoras es el ejercicio práctico de la resolución de problemas. Cuando se articula como juego en equipo, no importa la dificultad, porque todos los participantes emplearán sus recursos creativos para resolver el mismo. Recurrir a ejemplos prácticos sobre la resolución de problemas puede, inicialmente, generar dificultades, pero abre la puerta a nuevas perspectivas y contextos que ayuden a resolver dichos problemas. La escuela es el lugar idóneo para iniciar a los estudiantes en el proceso creativo, y la universidad, para la adquisición de estrategias que permitan la resolución de problemas basados en la creatividad.

Para estimular la creatividad en el aula, el discente desempeñará un rol activo, consciente y orientado hacia el cumplimiento de los objetivos del proceso, en interacción con los estudiantes. Para desplegar estos instrumentos, el docente debe tener capacidad educativa y formativa para favorecer la innovación creativa. Como sugiere Klimenko (2008), «la implicación en el proceso del juego creativo permite al estudiante convertir el proceso de aprendizaje relacionado con adquisición de destrezas y conocimientos necesarios en algo muy divertido y significativo a la vez» (p. 205).

A nuestro juicio, una buena metodología para potenciar la creatividad es aquella que tiene presente cuatro aspectos: modos de pensar, modos de trabajo, herramientas/instrumentos de trabajo y aspectos de vivir en sociedad.

\begin{abstract}
Recurrir a ejemplos prácticos sobre la resolución de problemas puede, inicialmente, generar dificultades, pero abre la puerta a nuevas perspectivas y contextos que ayuden a resolver dichos problemas
\end{abstract}

\section{Cuadro 1. Metodología para potenciar la creatividad}

Orientaciones

Modos de pensar

\section{Habilidades}

Creatividad e innovación.

Pensamiento crítico, resolución de problemas y situaciones sociales y toma de decisiones.

Aprender a pensar.

Conocimiento versus metaconocimiento.

Reflexividad, razonamiento y discernimiento. 


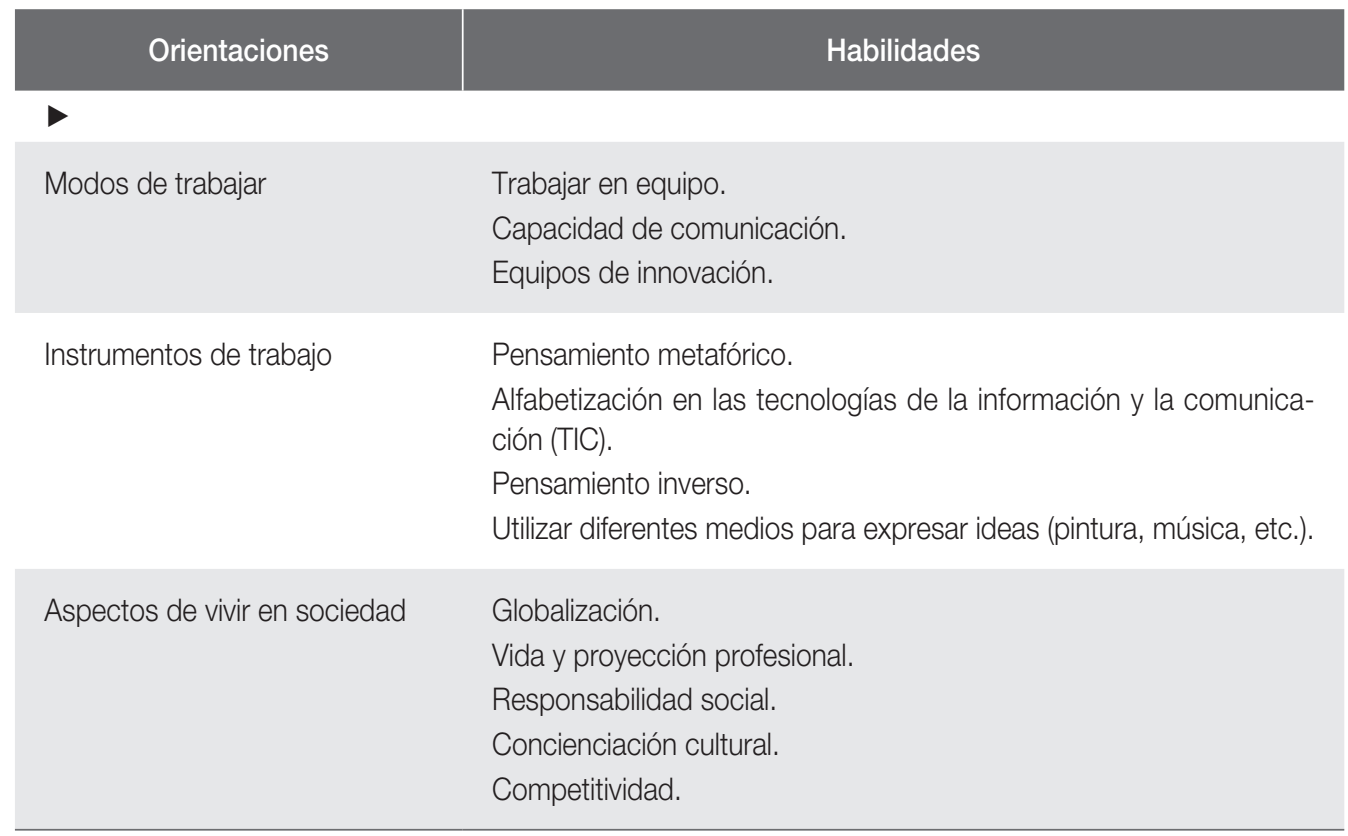

Fuente: Valero-Matas et al. (2016, p. 215).

En esa búsqueda de fomentar la creatividad en la docencia, el profesor debe reconocer el carácter transformador de su labor a partir de desarrollar en los estudiantes aquellos atributos más característicos de la creatividad, tales como la originalidad, la flexibilidad, la elaboración, la inventiva, la curiosidad, la sensibilidad e independencia. Lo específico de la creatividad en el ámbito docente educativo radica en la comprensión de las tareas y de los procedimientos de su solución, logrando la creatividad en los discentes y el establecimiento de ambientes que la propicien. La configuración de un escenario creativo en condiciones docentes exige poner a los estudiantes en situación de encontrar nuevas combinaciones y respuestas originales, partiendo de informaciones ya conocidas. Este propósito no se logra de forma improvisada. Para su estimulación y desarrollo han de interactuar muchos factores de índole diversa, por lo tanto, demanda un pensamiento flexible, dinámico, lateral, divergente, audaz e independiente; y este tipo de pensamiento no se desarrolla espontáneamente o al libre albedrío.

En este mismo orden, la creatividad profesional de un docente incluye la técnica en la especialidad, el contexto y el perfil de su objeto de trabajo, y lo cultural; y se expresa cuando el docente logra potenciar en los estudiantes la capacidad para afrontar y solucionar problemas en el desarrollo de su actividad práctica.

La creatividad profesional del docente se manifiesta cuando encuentra nuevos procedimientos, genera ambientes creativos que propician la creación individual o colectiva en el discente, así como el desarrollo de nuevas ideas teórico-prácticas. En el contexto educativo, la creativi- 
dad no debe ser lo excepcional, sino lo cotidiano, y constituye una expresión de profesionalidad en el área específica de desempeño donde se practique. Es una garantía para la mejora del rendimiento de los discentes desde las condiciones del aula. No existe creatividad docente sin expresión profesional, sin trabajador competente, sin servicio de calidad. El docente creador se replantea lo que hace diariamente; lo modifica constantemente, hasta conseguir un resultado final perfecto.
En el contexto educativo, la creatividad no debe ser lo excepcional, sino lo cotidiano. No existe creatividad docente sin expresión profesional, sin trabajador competente, sin servicio de calidad. El docente creador se plantea lo que hace diariamente

\subsection{Algunas indicaciones metodológicas para desarrollar la creatividad desde la educación}

\section{La mayor parte del aprendizaje con métodos creativos se desarrolla fuera del aprendizaje formal}

El primer punto de partida hacia la instrumentalización metodológica de la creatividad en el ámbito escolar es tener presente que la creatividad no sigue el modelo tradicional de enseñanza directa, sino que se fomenta, desarrolla y activa recurriendo a múltiples secuencias. La mayor parte del aprendizaje con métodos creativos se desarrolla fuera del aprendizaje formal, es decir, recurriendo al aprendizaje no formal o informal ejecutado por consultoras o compañías especializadas en métodos creativos para la resolución de problemas. Clifford (2016) considera que las escuelas mantienen vivo el modelo estandarizado de enseñanza y que son las instituciones educativas las que más lentamente adoptan la innovación. Según este autor son pocos los centros que han incorporado en sus programas la resolución creativa de problemas.

La educación creativa desde sus discursos abre muchas posibilidades a docentes y educadores para fomentar y desarrollar la creatividad en el aula (o espacios adecuados a ello). Algunas propuestas pueden ser:

- Acoger la creatividad como parte del aprendizaje. Establecer un espacio en el aula destinado a la creatividad, como diseño de tableros de anuncios para mostrar diferentes formas de resolver un problema o dar soluciones creativas en un escenario del mundo real.

- Usar las estrategias más efectivas. Torrance (2003) realizó un extenso metaanálisis sobre las formas más efectivas de enseñar la creatividad. Descubrió que las estrategias más exitosas en materia creativa empleaban el arte en todas sus dimensiones o programas orientados al uso o manejo de técnicas artísticas. 
- Pensar en la creatividad como una habilidad. Como ocurre con el ingenio y la inventiva, la creatividad es más un dominio que un rasgo y, por lo tanto, se puede enseñar. Al percibirlo de esta forma, el educador o formador debe encontrar la manera de potenciar esa habilidad creativa, dividirla en conjuntos e ir adiestrando al individuo en cada una de las secciones. En otras palabras, ir entrenando a la persona en cada una de las habilidades hasta alcanzar la destreza creativa.

- Participar o crear un programa para desarrollar habilidades creativas.

- Emplear conexiones emocionales. Utilizar programas que faciliten e introduzcan el elemento emocional en la actividad docente.

- Valorar el hecho de incluir en las tareas del aula el uso del pensamiento divergente y convergente. Las pruebas estandarizadas hacen un gran trabajo al medir el pensamiento convergente, que incluye pensamiento analítico o respuestas lógicas con una respuesta correcta. El pensamiento divergente considera el modo en que un alumno puede usar diferentes formas de abordar un problema. Requiere usar asociación y multiplicidad de pensamiento, por lo tanto, se deberían diseñar evaluaciones que consideren ambos tipos de modelos de pensamiento.

- La creatividad emerge en un «ambiente agradable». El pensamiento creativo necesita ser compartido y validado por otros en un ambiente de apoyo social. Cómo crear comunidades que fomenten la creatividad social para resolver problemas.

- Ver la creatividad como algo positivo. Enseñar la creatividad conlleva estar convencido de sus beneficios. En consecuencia, recompensar a los estudiantes por pensar en los problemas de distintas maneras, reconociendo sus esfuerzos.

- Fomentar el esfuerzo. El docente debe hacer posible que el esfuerzo se convierta en un valor clave en el desarrollo del niño porque crea un hábito de constancia. Esto permitirá que el niño salga reforzado ante las adversidades por su propia capacidad de superación. Si comete errores, ese empeño le llevará a intentarlo de nuevo.

- Aprender a tolerar la incertidumbre. El estudiante debe aprender a admitir la posibilidad de que un problema se puede resolver de muy diversas maneras. Es necesario que los estudiantes, tanto individual como colectivamente, reflexionen, porque lo que se aprende no es inmutable ni estático, todo lo contrario, el aprendizaje es dinámico. Es necesario animar a los discentes a explorar nuevas realidades; a salir de la cueva para explorar el universo.

- Fomentar la perseverancia para superar los obstáculos y las dificultades. Las adversidades están presentes en todas las esferas de la vida. La etapa educativa se inicia con un objetivo, que debe ser el aspecto prioritario, pero en la consecución de la meta aparecen infortunios que hay que aprender a superar.

- Desarrollar la confianza en sí mismo y en sus convicciones. Esto se consigue favoreciendo la apertura mental, la originalidad, asumiendo riesgos y planteándose preguntas, incluso cuestionando el conocimiento que se está desarrollando. 
- Desarrollar el pensamiento creativo y reflexivo. La escuela tiene el deber de hacer que los estudiantes desarrollen una cultura del trabajo tenaz, bajo la premisa de la recompensa intrínseca o extrínseca.

- Reconocer el potencial de los estudiantes y fomentar su desarrollo. Desarrollar el potencial de los estudiantes con el apoyo del docente para que en un futuro los propios discentes sepan extraerlo y fomentarlo.

- Dotar a los estudiantes de las herramientas necesarias para autoaprender en el modelo educativo enseñanza-aprendizaje. Los estudiantes necesitan conocer los instrumentos de aprendizaje y con ellos ejercitar las estrategias necesarias para lograr los objetivos propuestos.

\section{A modo de reflexión}

Existe una tendencia general en nuestra sociedad a pensar que las personas creativas son genios; que son personas hechas de otra materia. Pero la realidad ha demostrado que la creatividad se puede enseñar, desarrollar y potenciar si se utilizan los instrumentos adecuados para ello.

Los creadores no son meros expertos. La creatividad tiene una base de conocimiento profunda, y la práctica deliberada (Ericsson, 2008) puede contribuir a fomentar muchos aspectos de la creatividad. Pero, en última instancia, la creatividad implica mucho más que solo la práctica deliberada, como la predisposición o la capacidad innata, entre otros. Los creadores no son necesariamente los más eficientes, pero sus mentes desordenadas a menudo les permiten ver cosas que otros nunca verían y crear nuevos caminos que las generaciones futuras deliberadamente practicarán. El trabajo de Ericsson y Pool (2016) sobre la práctica deliberada es muy interesante, puesto que pensamos que dicha práctica deliberada puede ayudar a mejorar cualquier habilidad. Sin embargo, también creemos que una comprensión precisa de la creatividad es importante para la forma en que reconocemos, nutrimos, valoramos, etc., y, en última instancia, repercute y tiene un efecto positivo para la sociedad.

Realizar una tarea de forma virtuosa no se puede decir que sea producto de un hecho creativo. Por ejemplo, tocar un instrumento musical no es algo creativo si no suena una melodía; pintar el cuadro de Las meninas no es un hecho creativo, sino que es reproducir la creación de otro que sí ha sido creativo, etc. Esa idea de que todos somos creativos puede generar grandes fracasos y perturbar a los jóvenes. Diseñar modelos educativos basados en la creencia de que todos somos creativos, y hacerlo sin unos programas concretos, y tan solo abstractos, conlleva serios problemas. En todo lo enunciado a lo largo de este artículo se han escrito propuestas, pero ninguna de ellas se materializa sobre cómo llegar a la creatividad, es decir, son únicamente ideas. 
Como se puede apreciar, el desarrollo de la creatividad y de sus múltiples escenarios en la docencia no resulta tarea fácil. Sin embargo, de igual modo que el reto de su proyección se da por sentado, es ineludible la asunción de una orientación formativa que garantice niveles cualitativamente superiores de eficiencia académica, laboral y de investigación en la formación integral de los educandos; y en el perfeccionamiento constante de la labor docente en este ámbito.

Siguiendo las ideas de Robinson (2009) y los análisis de Amabile (2012) y Amstrong (2006), encontramos que probablemente uno de los desaciertos reside en que no se está diferenciando la enseñanza creativa de los modelos educativos de varias décadas atrás. Al igual que ocurre con la moda, se vuelve a los mismos diseños con nombres más modernos. En la educación actual ocurre algo similar, el lenguaje cambia, pero el contenido es el mismo de los años setenta y ochenta del siglo pasado, con una salvedad, el conocimiento. En aquellos años, el conocimiento dominaba la esfera del aprendizaje y sobre él pilotaba todo. Por entonces, los estudiantes formulaban preguntas interesantes, se cuestionaban si el reciclaje era una realidad o, simplemente, como se dice actualmente, un «postureo». Se enseñaba que el fracaso solo era posible cuando no se volvía a intentar. También se enseñaba lo que se ha dado en denominar «diferentes inteligencias», si bien no se impartía en profundidad en el colegio, sino en las actividades extraescolares. El ensayo y el error dominaban la actividad cotidiana, puesto que las prácticas eran la tónica. Además, se enseñaba a construir circuitos, casas, coches, etc.

Para poder reflexionar, el alumno debe saber si la reflexión se realiza desde una perspectiva crítica. El estudiante debe ser autónomo, sí, pero siempre ha de estar bajo el control del docente, porque, de lo contrario, para qué tener aulas; valdría con pequeños paraninfos en los que los estudiantes discutieran y generaran su propia educación. Entre las muchas cuestiones que hay que plantearse aparece una que debemos tener presente, y es la expresión que circula con fuerza en los sistemas educativos: ya no hay respuestas correctas.

Como hemos comentado anteriormente, pensar que todos somos creativos en mayor o menor medida puede ser peligroso. Se ha considerado que el problema de la creatividad residía en que no se potenciaba en la escuela esta área de conocimiento y que se iba relegando a un segundo plano a medida que el niño iba creciendo. Esta consideración de que la creatividad es un instrumento fundamental en el proceso de aprendizaje puede llevar a limitar otras capacidades si la creatividad se coloca en el punto más alto de la pirámide del proceso educativo. La creatividad es una capacidad más que hay que desarrollar dentro del juego creativo, pero no puede ser la capacidad última que hay que lograr en dicho juego. De lo contrario, seguiremos cayendo en los errores del pasado: focalizar el logro final en una capacidad, desatendiendo otras. 


\section{Referencias bibliográficas}

Amabile, T. (1996). Creativity in Context. Boulder, CO: West view Press.

Amabile, T. (2012). Componential Theory of Creativity. Boston: Harvard.

Armstrong, T. (2006). Inteligencias múltiples en el aula. Barcelona: Paidós.

Bono, E. de. (1999). El pensamiento creativo: el poder del pensamiento lateral para la creación de nuevas ideas. México: Paidós.

Clifford, S. (2016). Cultivating Creativity in Education, Nimega: Radboud University.

Csikszentmihalyi, M. (1998). Creatividad: el fluir y la psicología del descubrimiento y la invención. Barcelona: Paidós.

Csikszentmihalyi, M. (1999). Implications of a systems perspective for the study of creativity. En R. J. Sternberg (Ed.), Handbook of Creativity (pp. 16-34). Cambridge: Cambridge University Press.

Ericsson, K. A. (2008). Deliberate practice and the acquisition and maintenance of expert performance: a general overview. Academic Emergency Medicine, 15(11), 988-994. doi: 10.1111/j.1553-2712.2008.00227.x.

Ericsson, A. y Pool, R. (2016). Peak: Secrets from the New Science of Expertise. London: Bodley Head.

Ferrando, M., Ferrándiz, C., Bermejo, M. ${ }^{a}$ R., Sánchez, C., Parra, J. y Prieto, M. ${ }^{a}$ D. (2007). Estructura interna y baremación del test de pensamiento creativo de Torrance. Psicothema, 19(3), 489-496.

Gardner, H. (1987). Estructuras de la mente: la teoría de las múltiples inteligencias. México: Fondo de Cultura Económica.

Gardner, H. (1995). Mentes creativas: una anatomía de la creatividad. Barcelona: Paidós.

Gardner, H. (2011). Mentes líderes. Barcelona: Paidós.
Gardner, H. (2012). Inteligencias múltiples: la teoría en la práctica. Barcelona: Paidós.

Getzels, J. y Jackson, P. W. (1962). Creativity and Intelligence: Explorations with Gifted Students. New York: Wiley.

Guilford, J. P. (1950). Creativity. American Psychologist, 5(9), 444-454.

Guilford, J. P. (1988). Some changes in the structure of intellect model. Educational and Psychological Measurement, 48, 1-4.

Klimenko, O. (2008). La creatividad como un desafío para la educación del siglo XXI. Educación y Educadores, 11(2), 191-210.

Kris, E. (1952). Psychoanalytic Exploration in Art. New York: International University Press.

Landau, E. (1987). El vivir creativo: teoría y práctica de la creatividad. Barcelona: Herder.

Lowenfeld, V. (1989). El niño y su arte. Buenos Aires: Kapelusz.

Lowenfeld, V. (1992). Desarrollo de la capacidad creadora. Buenos Aires: Kapelusz.

Lowenfeld, V. y Lambert Brittain, W. (1947). Creative and Mental Growth. New York: Macmillan.

May, R. (1977). La valentía de crear. Buenos Aires: Emecé.

Maslow, A. (1994). La personalidad creadora. Barcelona: Kairós.

Mednick, S. (1962). The associative basis of the creative process. Psychological Review, 69, 220-232.

Parnes, S. (1967). Creative Behaviour Guidebook. New York: Charles Scribner's Sons.

Robinson, K. (2009). The Element: How Finding Your Passion Changes Everything. London: Viking. 
Torrance, E. P. (1965). Educación y capacidad creativa. Madrid: Marova.

Torrance, E. P. (2003). Orientación del talento creativo. Buenos Aires: Troquel.

Torrance, E. P. y Myers, R. (1996). La enseñanza creativa. Madrid: Santillana.

Torre, S. dela. (2009). Adversidad creadora: teoría y práctica del rescate de potenciales la- tentes. Revista Encuentros Multidisciplinares, 31, 6-20.

Valero-Matas, J. A., Valero-Oteo, I., Coca, J. R. y Leyva, A. L. (2016). Creatividad y educación para el siglo XXI desde una perspectiva sociológica. RIPS: Revista de Investigaciones Políticas y Sociológicas, 15(2), 201-222. doi: http://dx.doi.org/10.15304/rips.15.2. 3473.

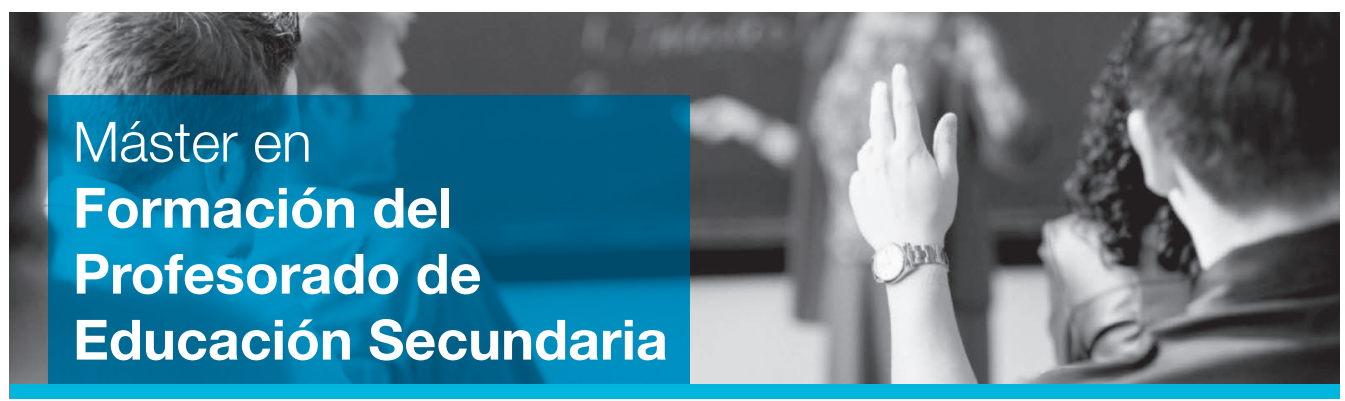

Este máster oficial en Formación del Profesorado de Educación Secundaria Obligatoria, Bachillerato, Formación Profesional y Enseñanza de Idiomas [60 créditos ECTS] tiene una duración normal de 12 meses.

Dirigido a: La universalización de la enseñanza secundaria y el incremento de la atención a la diversidad de alumnos en todos los niveles de enseñanza han hecho más patente la necesidad de mayor formación didáctica. El educador ya no solo ha de ser un experto en su materia, sino que debe tener la suficiente capacidad didáctica para adaptar la misma a grupos de alumnos muy heterogéneos en intereses, capacidades y actitudes.

Objetivos: Adquirir todas las habilidades y competencias necesarias para poder desarrollar una carrera profesional en el ámbito de la enseñanza en los niveles de Educación Secundaria Obligatoria y Bachillerato, Formación Profesional y Enseñanzas de Idiomas de acuerdo a la normativa vigente, ya sea para dar clase en centros públicos, privados o concertados. Ofrecer formación integral y especializada a los participantes. 